\title{
IDENTIFICACIÓN DE COMPETENCIAS Y CARACTERÍSTICAS DESEABLES EN EL PROFESORADO DE CIENCIAS DE EGB
}

\author{
RODRIGO, M., AGRA.CADARSO, M.J., GÓMEZ, M.A., MORCILLO, J.G., \\ UNAMUNO, M. Y VIDAL, M.P. \\ Departamento de Didactica de las Ciencias Experimentales. Universidad Complutense. Madrid. \\ EU María Díaz Jiménez. Departamento de Didáctica de las Ciencias. Av. de Filipinas, 3. 28003 Madrid.
}

\section{SUMMARY}

This article offers the vision held by some groups of teachers on various qualities taken as important for a Science Teacher of Primary Education, as well as the opinion on to what extend those qualities are found in this type of teachers.

\section{INTRODUCCIÓN}

El profesor es considerado como un agente capital en los procesos de innovación educativa (Escudero 1986). Por otro lado, cada vez se avanza más en el paradigma del profesor reflexivo en la acción (Pérez Gómez 1987, Villar Angulo 1992), y se insiste en la inseparable unión de teorías personales del profesorado y su quehacer didáctico (Kagan 1990); también en el campo de la enseñanza de las Ciencias (Brickhouse 1990, Cornet et al. 1990, Lucas 1986, Roberts y Chastko 1990).

Según todo ello, el presente trabajo se centraría en la preguntasiguiente: ¿Cuáles son las creencias del profesorado de Ciencias de EGB sobre determinadas variables o competencias que de acuerdo con la investigación didáctica (Brincones et al. 1986, Hewson y Hewson 1988) parecen incidir en la calidad de la enseñanza en este campo?

La citada cuestión, en estos momentos de Reforma Educativa en España, creemos que es esencial para poder abordar acciones encaminadas hacia la formación inicial y permanenete del profesorado de Ciencias en la enseñanza secundaria obligatòria sobre la que incide esta investigación.

\section{METODQLOGÍA Y TÉCNICAS DE INVES- TIGACIÓN. ESTUDIOS PREVIOS SOBRE EL TEMA}

En la bibliografía consultada no hemos encontrado estudios semejantes al que aquí presentamos para profesores de Ciencias de EGB en España. Existen sólo algunos estudios generales sobre la situación de la formación inicial del profesorado (Albuerne et al. 1986, Pérez Serrano 1988) y más concretos sobre el pensamiento del profesorado de Ciencias y de sus alumnos en niveles de bachillerato (Brincones et al. 1986, Carrascosa et al. 1991).

\section{EL INSTRUMENTO DE RECOGIDA DE DATOS}

Se utilizará el procedimiento de sondeo de opinión a través de cuestionario.

El cuestionario piloto inicial surge de las aportaciones procedentes de la revisión de la bibliografía nacional y extranjera. En concreto, siguiendo la propuesta de Nieda 
et al. (1988), elegimos 23 variables de estudio que se clasifican en cuatro apartados:

- Competencias relacionadas con la materia.

- Competencias relacionadas con las técnicas de enseñanza.

- Características personales del profesor.

- Características profesionales del profesor.

Después de un proceso de maduración y discusión, así como una elemental fase de preencuesta a un grupo de die $z$ profesores de Ciencias de EGB, se ha elaborado un "cuestionario» que presenta ligeras modificaciones del propuesto por Nieda en 1988. (Véase en el Apéndice). Recordemos aquí que se solicita en cada competencia una valoración entre cuatro categorias (no deseable, indiferente, importante, esencial) y una estimación de posesión entre cuatro categorías (prácticamente ninguno, pocos, bastantes, prácticamente todos).

\section{SELECCIÔN DE MUESTRAS DE ESTUDIO}

a) Poblaciôn bấsica de estudio: profesores de Ciencias de EGB do Madrid. (Población normal, Ml).
A partir del documento editado por el MEC en 1990 sobre la "Catalogación de puestos de docentes», se ha confeccionado una relación de profesores de Ciencias del ciclo superior de EGB de Madrid (Véanse los centros seleccionados en Rodrigo et al. 1992 b).

El número de profesores de Ciencias de EGB en los centros públicos de Madrid y la relación numérica de la muestra prevista fue la siguiente:

Número de profesores de Ciencias de EGB ...... 1.755

Número de profesores a encuestar

b) Otra población de referencia. (Población específica, M2).

Éstos son profesores de EGB que «voluntariamente» han recibido cursos de «Actualización Científica y Didáctica en Ciencias» promovidos por el MEC, en 1989 1990.

En este caso, según información procedente de la Subdirección General de Formación del Profesorado, se tenía previsto realizar el «curso" en las demarcaciones de Ávila, Zaragoza y Cáceres. Aunque en esta última no se ha realizado, sí se ha llevado a cabo en las otras dos provincias. Puestos al habla con los directores de los

Tabja I

Porcentajes de encuestados que consideran las distintas competencias profesionales como «esenciales» o como cojuntamente «importantes o ensenciales» tanto para la población M 1 o normal $(n=98)$ y la $M 2$ o específicamente formada durante un año $(n=32)$.

\begin{tabular}{|c|c|c|c|c|}
\hline Datos X & Esencial - M1 & Esencial - M2 & Impor+Esen-M1 & Impor+Esen-M2 \\
\hline 1 Conocer la disciplina & 33,7 & 56,3 & 100 & 100 \\
\hline 2 Ensenar inplicaciones & 31,6 & 40,6 & 97,9 & 100 \\
\hline 3 Enseñanza interdisciplinaria & 35,7 & 37,5 & 95,9 & 96,9 \\
\hline 4 Relaciona la ciencia & 48 & 59.4 & 96 & 96,9 \\
\hline 5 Ensena hist. y fil de la ciencia & 12,2 & 12,5 & 82,6 & 87,5 \\
\hline 6 Enseña métodos & 62,2 & 68,8 & 97,9 & 96,9 \\
\hline 7 Prepara y organiza & 48 & 43,8 & 99 & 96,9 \\
\hline 8 Planifica enseñanza & 48 & 50 & 94,9 & 93,8 \\
\hline 9 Prepara las ideas & 54,1 & 40.6 & 97 & 100 \\
\hline 10 Conoce y usa psicología & 31,6 & 40,6 & 86,7 & 93,7 \\
\hline 11 Usa métodos activos & 48 & 37,5 & 94,9 & 100 \\
\hline 12 Crea un clima humano & 66,3 & 56,3 & 97,9 & 100 \\
\hline 13 Usa recursos y técnicas & 24,5 & 37,5 & 93,9 & 81,3 \\
\hline 14 Enseña de maneza ind. & 16,3 & 25 & 79,6 & 71,9 \\
\hline 15 Evałlia adecuadamente & 32,7 & 50 & 94,9 & 93,8 \\
\hline 16 Usa los resultados & 35,7 & 40,6 & 90,8 & 90,6 \\
\hline 17 Tiene estabilidad & 31,6 & 53,1 & 94,9 & 93,7 \\
\hline 18 Tiene sentido del humor & 14,3 & 28,1 & 76,5 & 75 \\
\hline 19 Muestra entusiasmo & 38,8 & 46.9 & 93,9 & 96,9 \\
\hline 20 Se relaciona cordialmente & 18,4 & 25 & 85,7 & 75 \\
\hline 21 Es autocrítico & 39,8 & 40,6 & 96,9 & 96,9 \\
\hline 22 Coopera con otros & 31,6 & 40,6 & 90,8 & 93,7 \\
\hline 23 Se perfecciona & 25,5 & 43,7 & 93,9 & 100 \\
\hline
\end{tabular}


cursos, hemos contabilizado una población de 22 profesores de EGB en Ávila y 18 profesores de EGB en Zaragoza.

\section{EL TRABAJO DE CAMPO}

EI grueso de esta fase, es decir, la realización de las encuestas, se realizó durante el $2^{\circ}$ trimestre del curso escolar 1989-90. En el caso de la Población Normal (M1) se recogieron 98 encuestas (es de destacar que en este caso el número de respuestas obtenidas ha sido menor del esperado, aspecto que deberemos tener presente en la discusión global de resultados). En el caso de la «Población» específicamente formada (M2) se recogieron 32 encuestas (es decir, un altísimo porcentaje).

\section{ANÁLISIS DE LOS DATOS OBTENIDOS}

Estos análisis se han realizado con paquetes estadísticos como el BMDP, que autores como Neida y otros (1988) han utilizado en estudios semejantes. Se han Ilevado a cabo durante el curso $1991-92$ en el Centro de Proceso de Datos de Ia Universidad Complutense de Madrid.

\section{PRESENTACIÓN DE RESULTADOS. IMPORTANCIA DE COMPORTAMIENTOS Y CARACTERISTICAS}

Según se desprende de los datos ofrecidos en la tabla I, son valoradas en grado alto (IMP+ESENCIAL) prácticamente todas la competencias estudiadas tanto en la población M1 como en la M2.

Si en la tabla I nos fijamos exclusivamente en el porcentaje de encuestados que consideran «esenciales» diversas competencias, tendríamos:

a) Tienden a ser consideradas como esenciales, por más del $50 \%$, las competencias:

* 6 (62,2\% para M1 y $68,8 \%$ para M2): Enseñar los métodos de la Ciencia.

* $12(66,3 \%$ y $56,3 \%$ respectivamente): Crear un clima humano entre los alumnos que ayude a aprender.

* 4 (48\% y $59 \%$ respectivamente): Enseñar las Ciencias como cercanas.

* $8(48 \%$ y $50 \%$ respectivamente): Planificar la enseก̃anza.

b) Por menos del $25 \%$, las competencias:

* 5 ( $12,2 \%$ y $12,5 \%$ respectivamente): Enseñar la historia y la filosofía de la ciencia.

* 14 (14,3\% y $25 \%$ respectivamente): Enseñar de manera individualizada.

* $18(14,3 \%$ y $28,1 \%$ respectivamente): Tener sentido del humor.

* 20 ( $18,4 \%$ y $25 \%$ respectivamente): Relacionarse cordialmente con padres y alumnos. c) En general no se notan grandes diferencias en la valoración de las competencias entre la población Ml y M2 aunque los profesores de esta última tienden, en mayor porcentaje, a catalogar como esenciales las características estudiadas. En particular destaca la diferencia de «esencialidad» dado al conocimiento de la materia; así sería para un $33,7 \%$ de los profesores M1 frente a un $56,3 \%$ de los profesores M2.

\section{GRADO DE MANIFESTACIÓN DE LOS COM- PORTAMIENTOS Y CARACTERÍSTICAS}

\author{
Tabla II
}

\begin{abstract}
Porcentajes de encuestados que consideran presentes las distintas competencias en «bastantes» o en «prácticamente todos» los profesionales. $\mathrm{MI}=$ Datos de profesores normales $(\mathrm{n}=98)$ y $\mathrm{M} 2=$ Datos de profesores especificamente formados durante ur año $(n=32)$
\end{abstract}

Datos X

Bast + Ptodos - MI

Bast + Ptodos - M2

$\begin{array}{lll}\text { 1 Conocer la disciplina } & 50 & 46,9 \\ \text { 2 Enseñar implicaciones } & 58,2 & 56,3 \\ \text { 3 Enseñanza interdisciplinaria } & 39,8 & 43,8 \\ \text { 4 Relaciona la ciencia } & 49 & 59,4 \\ \text { S Enseña hist. y fil. de la ciencia } & 42,9 & 21,9 \\ \text { 6 Enseña métodos } & 49,9 & 40,6 \\ \text { 7 Prepara y organiza } & 30,6 & 31,3 \\ \text { 8 Planifica enseñanza } & 72,4 & 78,1 \\ \text { 9 Prepara las ideas } & 68,4 & 62,5 \\ \text { 10 Conoce y usa psicología } & 27,6 & 28,1 \\ \text { I1 Usa métodos activos } & 54,1 & 40,6 \\ \text { 12 Crea un clima humano } & 78,6 & 84,4 \\ \text { I3 Usa recursos y técnicas } & 44,9 & 43,8 \\ \text { 14 Enseña de manera ind. } & 22,4 & 31,3 \\ \text { 15 Evalúa adecuadamente } & 63,3 & 46,9 \\ \text { I6 Usa los resultados } & 43,9 & 31,3 \\ \text { 17 Tiene estabilidad } & 48 & 56,3 \\ \text { 18 Tiene sentido del humor } & 52 & 46,9 \\ \text { 19 Muestra entusiasmo } & 50 & 78,1 \\ \text { 20 Se relaciona cordialmente } & 63,3 & 62,5 \\ \text { 21 Es autocrítico } & 44,9 & 25 \\ \text { 22 Coopera con otros } & 31,6 & 28,1 \\ \text { 23 Se perfecciona } & 23,5 & 37,5\end{array}$

Según se desprende de los datos ofrecidos en la tabla II, referidos al porcentaje de encuestados que estiman el grado de posesión de las distintas competencias, podemos considerar:

a) Más del $75 \%$ (de media entre M1 y M2) de los encuestados consideran muy extendidas las competencias:

* $12(78,6 \%$ en la población M1 y $84,4 \%$ en la M2): Crear un clima humano entre los alumnos que ayude a aprender.

* $8(72,4 \%$ en la M1 y $78,1 \%$ en la M2): Planificar la enseñanza.

b) Menos del 35\% (de media entre M1 y M2) de los encuestados consideran muy extendidas las competencias: 
* 5 (42,9\% para M1 y 21,9\% para M2): Enseña filosofía e historia de la ciencia.

* 7 (30,6\% para M1 y 31,3\% para M2): Preparar y organizar adecuadamente el trabajo de laboratorio.

* $10(27,6 \%$ para M1 y $28,1 \%$ para M2): Tener y usar conocimientos de psicología del aprendizaje.

* 14 (22,4\% para M1 y 31,3\% para M2): Enseñar de manera individualizada.

* 21 (44,9\% para MI y $25 \%$ para M2): Ser autocrítico.

* 22 (31,6\% para la M1 y $28,1 \%$ para M2): Cooperar con otros profesores.

* $23(23,5 \%$ para M1 y 37,5\% para M2): Perfeccionarse científica y pedagógicamente.

c) En general no se notan grandes diferencias de «apreciación de posesión de competencias» entre la población M1 y M2.

RELACIÓN ENTRE LA IMPORTANCIA CONCEDIDA Y LOS GRADOS DE MANIFESTACIÓN ESTIMADOS PARA LAS COMPETENCIAS ESTUDIADAS

Figura 1

Distribución de porcentajes de encuestados que valoran como «esencial» las diversas competencias y porcentajes de los que las estiman como "muy extendidas" (Bast+Ptodos) entre el profesorado.

(Datos referidos a la población Ml o de profesores normales, $\mathrm{n}=98$ ),

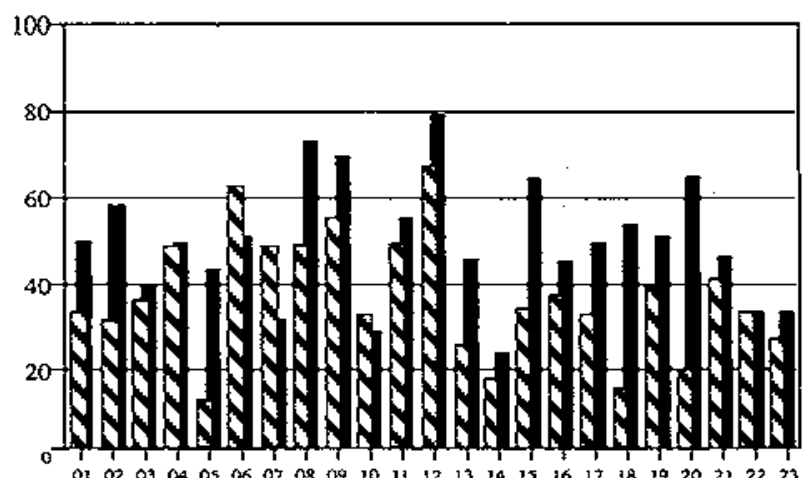

WESENCIALM-1 Bast+PTODOS-MI

De las tablas I y II de las figuras 1 y 2 , podemos sacar las siguientes observaciones.

a) Son consideradas conjuntamente como esenciales por un alto porcentaje de encuestados (superior al $50 \%$ ) y como muy extendidas por un gran número de encuestados (superior al $75 \%$ ) las competencias:

12. Crear un clima humano entre los alumnos que ayuden a aprender.

\section{Planifícar la enseñanza.}

b) Existe poco acuerdo en su extensión entre el profesorado de otras competencias que si fueron consideradas como esenciales en un porcentaje superior al $50 \%$ como:
Figura 2

Distribución de porcentajes de encuestados que valoran como "esencial» las diversas competencias y porcentajes de los que las estiman como "muy extendidas» (Bast+Ptodos) entre el profesorado. (Datos referidos a la población M2 o de profesores específicamente formados durante $I$ año, $\mathrm{n}=32$ ).

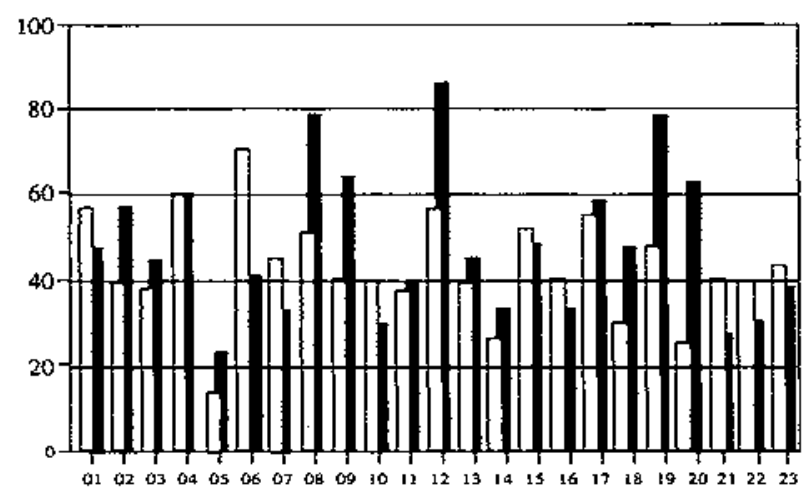

ESENCIAL-M2 BAST+PTODOS-M2

\section{Enseñar las ciencias como cercanas. \\ 6. Enseñar los métodos de la ciencia.}

c) Entre las competencias consideradas como muy poco extendidas entre el profesorado (menos del $35 \%$ de los encuestados las consideran presentes) y que son valoradas como esenciales por un porcentaje cercano al $50 \%$, destacarían las características:

21. Ser autocrítico, reconocer sus limitaciones y evaluarse a sí mismo.

22. Coopera con otros profesores.

23. Perfeccionarse científica y pedagógicamente.

\section{DISCUSIÓN}

Importancia de comportamientos y características

En primer lugar destaca que prácticamente todas las competencias sean valoradas como importantes para la mayoría de los profesores tanto de la población M1 como de la M2.

Este hecho podría deberse a:

- Que no se trata de variables aleatorias sino que han sido seleccionadas por su importancia para la didắctica de las ciencias.

- Que su formulación es general (a pesar de acompañarse con ejemplos, véase el apendice) y da cierta oportuni- 
dad al profesorado para mostrar sus respuestas desde una visión optimista.

Por otro lado, en estudios similares con profesores e inspectores de bachillerato, Nieda y otros, en 1988, también reflejan esta circustancia. Tal hecho motivó en ellos tomar los porcentajes de respuestas en el apartado «esencial» como el criterio que podría defínir la valoración de importancia. Tal criterio, lo asumimos aquí y así permitirá una mejor constrastación de datos.

Manteniendo el criterio indicado, al igual que Nieda y otros (1988), observamos en algunas respuestas una cierta ausencia de bases objetivas sobre las valoraciones de los encuestados. Así, por ejemplo, destaca el que la competencia 12 (Crear un clima humano entre los alumnos que ayude a aprender) sea valorada como esencial por porcentajes superiores al $60 \%$ de los entrevistados; sin embargo la competencia 14 (Enseñar de una manera individualizada), que implicaría de alguna manera a la anterior, es valorada como esencial por menos de $25 \%$ de los entrevistados. No obstante, esto no quita que este estudio, en términos globales, nos sirva para reflejar preocupaciones y valoraciones que presenta el profésorado y que creemos que son muy interesantes a la hora de abordar estos momentos educativos, como seguidamente veremos.

De los datos de valoración de importancia, destacan la altísima valoración (porcentajes en torno al $65 \%$ ) de los que piensan que son esenciales las competencias 12 (Crear un clima...) y 6 (Enseñar los métodos de la ciencia). Precisamente esta última competencia es citada como la más valorada en estudios de autores como Nieda y otros (1988), en España, y Chiapetta y Collete (1978 a y b), en EEUU, pero con profesores e inspectores de enseñanza secundaria. Sin embargo, la competencia 12 no presenta este altísimo índice de valoración en esos estudios citados, y en cambio es reffejada como de las más esenciales en autores como Anderson (1982) y Yager y Penick (1984), e incluso como la más esencial en estudios llevados a cabo con profesores de ciencias de EGB en formación inicial (Rodrigo et al. 1992a). (Véase la figura 3 en la que quedan representados los porcentajes de valoración esencial dados por 212 profesores en formación inicial.)

También destaca la alta valoración de la competencia 8 (Planificar la enseñanza), que en similares estudios sobre el pensamiento del profesorado de ciencias (Nieda et al. 1988 y Chiapetta y Collette 1978 a y b) quedó manifestada como de máxima trascendencia.

Otro dato destacable sería la escasa esencialidad (por debajo de un $13 \%$ de los encuestados to cree así) de la competencia 5 (Enseñar la historia y la filosofía de la ciencia). Este dato está en la línea de los obtenidos en los estudios ya citados de Nieda y otros (1988); de Carrascosa y otros (1991), que estudian el pensamiento de alumnos de bachillerato, y de Rodrigo y otros (1992 a), que estudian el pensamiento del profesor de ciencias de EGB en formación inicial (Fig. 3). Sin duda esto contrasta (igual que indicaron en $1988 \mathrm{Nieda}$ y otros) con la alta valoración que se ofrece para la competencia 6 (Enseñar
Ios métodos de la ciencia), lo que podría indicar que estos profesores estudiados no han asumido el qué, cómo y cuándo de la implantación de este principio de innovación, principio que para muchos (Pessoa de Carvalho 1991) es importante para mejorar la enseñanza de las ciencias.

\section{Figura 3}

Tantos por ciento referidos a la valoración máxima o esencial obtenidos por cada competencia al estudiar el pensamiento de futuros profesores de ciencias en formación inicial (Rodrigo et al. 1992a).

CARACTERÍSTICA ESENCIAL

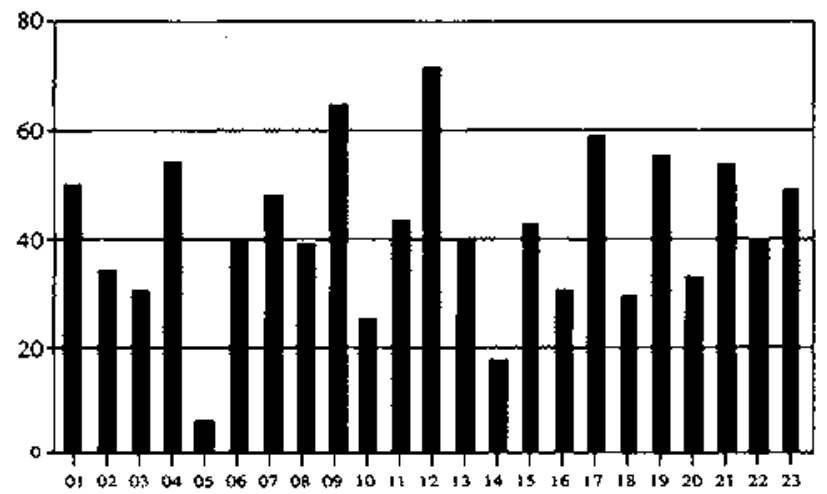

ESCUELAS A+B

Por último, y como dato curioso, debemos destacar que a pesar de la gran coincidencia entre las valoraciones formuladas tanto por profesores M1 como M2 en alguna competencia como la 1 (conocer las disciplinas del área) se da una fuerte discrepancia. Así es valorada como esencial por el 56,3\% de los profesores M2 (o profesores fornados en didáctica de las ciencias durante un curso escolar) frente a un 33,7\% de los profesores Ml. La importancia esencial de que el profesor conozca la materia a enseñar ha sido puesta de manifiesto como una cualidad imprescindible por los inspectores de bachille. rato entrevistados por Nieda y otros (1988), por Ios alumnos de bachillerato investigados por Carrascosa y otros (1991), y es caracterizada como tal por autores como Gil Pérez (1991).

\section{Manifestación de las competencias y características:} Implicaciones en la formación del profesorado

En la línea de lo que ya se ha expresado al discutir las valoraciones podemos indicar que:

- No se notan grandes diferencias entre las opiniones relativas a la extensión de cada competencia entre los profesores M1 con respecto a las de M2.

- En algunas opiniones se nota una cierta ausencia de objetividad. Así por ejemplo, se cataloga la competencia 8 (Planificar la enseñanza) como muy extendida entre el profesorado; en cambio la 7 (Preparar y organizar ade- 
cuadamente el trabajo de laboratorio) se presenta como muy poco extendida a pesar de su gran relación con la anterior.

No obstante, como vamos a ver a continuación los datos que discutiremos son, cuando menos, esclarecedores de posibles campos de actuación en la formación del profesorado.

Así, si tomamos como referencia la alta valoración de esencialidad de las competencias 12 (Crear un clima...) y 8 (Planificar la enseñanza) unida a la alta estimación de presencia entre el profesorado (más del $75 \%$ del profesorado estudiado creen que una gran mayoría las tiene), parecería que al menos en este grupo de competencias poco se debería mejorar en la formación. Estos datos, podemos contrastarlos con los ofrecidos por inspectores de bachillerato en el estudio de Nieda y otros (1988); así, existe coincidencia en cuanto a la competencia 12 , pero no en la 8, que, a pesar de ser catalogada como muy esencial, es estimada como muy poco extendida entre el profesorado de ciencias de bachillerato. Aquí deberíamos recordar que nuestro trabajo se ha centrado en profesores de EGB y, por lo tanto, creemos que se debería profundizar en este apartado en próximas investigaciones. A pesar de esta discrepancia, el trabajo ofrecido por Nieda y otros (1988) ya avanzaba posiblescarencias en la formación del profesorado investigado, que también se encuentran en nuestro estudio y que se centrarian en:

a) Competencias metodológicas (que además son valoradas en nuestro estudio como esenciales por encima del $50 \%$ del profesorado) como:

* 6. (Enseñar los métodos de la ciencia).

* 7. (Preparar y organizar adecuadamente el trabajo de laboratorio).

b) Competencias actitudinales (que son valoradas en nuestro estudio como esenciales por porcentajes cercanos al $50 \%$ del profesorado) como:

* 21 (Ser autocrítico).

* 22 (Cooperar con otros profesores).

* 23 (Perfeccionarse científica y pedagógicamente).

c) Competencias de innovación, que, a pesar de la cultura pedagógica imperante e incluso a pesar de ser parte de principios esenciales de la Reforma Educativa del MEC (1989), son catalogadas como esenciales por una minoría del profesorado encuestado, como son:

* 5 (Enseñar la historia y filosofía de la ciencia).

* 10 (Tener y usar conocimientos de psicología del aprendizaje).

* 14 (Enseñar de una manera individualizada).

\section{CONCLUSIONES}

A. Todas las competencias estudiadas son valoradas como importantes por la gran mayoría del profesorado estudiado tanto del grupo normal (Población M1) como del grupo específicamente formado durante un año (Población M2), lo cual nos ha motivado a tomar porcentajes de respuestas en el apartado esencial como referencia para la evaluación de este tipo de respuestas. Un aspecto similar se dio estudiando el pensamiento del profesor de ciencias en formación inicial (Rodrigo et al. 1992a).

B. Las valoraciones de las diversas competencias ofrecidas por los profesores MI son, en términos generales, similares a las de los $\mathrm{M} 2$, así mismo las estimaciones de posesión de las distintas competencias. No obstante, algunas respuestas del estudio manifiestan cierta ausencia de objetividad, to que implica que las conclusiones que se establecen sean tenidas como referencias importantes del pesamiento del profesorado estudiado pero no excluyentes de otras posibles investigaciones.

C. Han sido estimadas como poco corrientes y valoradas, como bastante esenciales los siguientes grupos de competencias:

a) Las claramente relacionadas con el área de didáctica de las ciencias, como son:

- 6. (Enseñar los métodos de la ciencia).

- 7. (Preparar y organizar adecuadamente el trabajo de laboratorio).

b) O bien otras claramente relacionadas con las actitudes personales y profesionales como:

-21 . (Ser autocrítico).

- 22. (Cooperar con otros profesores).

- 23: (Perfeccionarse científica y pedagógicamente).

A la luz de esto se podría pensar que deberian tenerse presentes estos dos campos de actuación en la formación y perfeccionamiento del profesorado.

D. Por otra parte, destaca singularmente la importancia, y también el alto grado de posesión, entre el profesorado, de la comptetencia 12 (Crear un clima humano entre los alumnos que ayude a aprender). Por el contrario, destacaría por su poca aparente importancia $y$, así mismo escaso grado de posesión entre el profesorado, de las competencias 5 (Enseñar la historia y la filosofía de la ciencia) y la 14 (Enseñar de manera individualizada), a pesar de la cultura didáctica imperante en este campo. Esta última conclusión, en lo referente a la valoración de importancia, está en la línea de lo observado por nosotros con profesores de ciencias en formación inicial. (Véase la figura 3 y Rođrigo et al. 1992a.)

\section{AGRADECIMIENTOS}

Este trabajo es parte de un proyecto de investigación financiado por la Universidad Complutense de Madrid. Agradecemos los consejos y orientaciones de las profesoras J. Nieda e I. Fuentes así como los del profesor J. Otero, que fueron los pioneros de este tipo de investigaciones en España. Por último, nuestro más sincero agradecimiento a todos Ios profesores y a los directores de los centros de EGB que consiguieron que esta investigación fuera posible. 


\section{REFERENCIAS BIBLIOGRÁFICAS}

ALBUERNE, F., GARCÍA, G. y RODRÍGUEZ ROJO, M., 1986. Las Escuelas Universitarias de Magisterio: Análisis y alternativas. (ICE: Universidad de Oviedo).

ANDERSON, C.S., 1982. The search for School climate: A review of the research, Review of Educational Research, Vol. 52 (3) pp. 368-420.

BRICKHOUSE, N.W., 1990. Teachers'Beliefs about the Nature of Science and their Relationship to Classroom Practice, Journal of Teacher Education, Vol. 41 (3), pp. 53-62.

BRINCONES, I., FUENTES, A., NIEDA, J., PALACIOS, M.J. y OTERO, J., 1986. Identificación de comportamientos deseables del profesorado de Ciencias Experimentales del Bachillerato, Enseñanza de las Ciencias, Vol. 4(3), pp. 209-222.

CARRASCOSA, J., FERNÁNDEZ, I, y OROZCO, A., 1991. La visión de los alumnos sobre lo que el profesorado de Ciencias ha de saber y saber hacer, Investigación en la Escuela, Vol. 14, pp. 44-61.

CHIAPETTA, E.C. y COLLETTE, A.T., 1978a. Secondary Science Teacher Skills Identified by Science Supervisors, Science Education, Vol. 62 (1), pp. 67-71.

CHIAPETTA, E.C. y COLLETTE, A.T., 1978b. Secondary Science Teacher $\$$ kills Identified by Secondary Teachers, Science Education, Vol. 62 (1), pp. 73-78.

CORNETT, J.W., YEOTIS, C. y TERWILLIGER, L., 1990. Teacher Personal Practical Theories and their Influence upon Teacher Curricular and Instructional Actions: A Care Study of a Secondary Science Teacher, Science Education, Vol. 74 (5), pp. 517-529.

ESCUDERO, J.M., 1986. EI pensamiento del profesor y la innovación educativa, en Villar, L.M. (ed.), Pensamiento de los profesores y toma de decisiones, pp. 7-36. (GID: Sevilla).

GIL PÉREZ, D., 1991. ¿Qué hemos de saber y saber hacer los profesores de Ciencias?, Enseñanza de las Ciencias, Vol. 9 (1), pp. 69-77

HEWSON, P.W. y HEWSON, G.N., 1988. An Approprate conception of teaching Science: A view from studies of sctence learning, Science Education, Vol. 72(2), pp. $597-614$.

KAGAN, D.M., 1990. Ways of Evaluating Teacher cognition: Inferences Concerning the Groldilocks Principle, Review of Educational Review, Vol. 60(3), pp. 419-469.
LUCAS, A.M., 1986. Tendencias en la investigación sobre la enseftanza aprendizaje de la Biología, Enseñanza de las Ciencias, vol. 4(3), pp., 189-198.

MEC, 1989. Libro Blanco para la Reforma del Sistema Educativo. (MEC: Madrid).

MEC, 1990. Relación de puestos de trabajo en los centros públicos de Preescolar, EGB y Educación Especial, Comunidad Escolar, núm, extra.

NIEDA, J., BRINCONES, I., FUENTES, A., OTERO, J. y PALACIOS, J.M., 1988. Identificación de comportamientos y características deseables del profesor de Ciencias Experimentales del Bachillerato. (CIDE: Madrid).

PÉREZ GÓMEZ, A.I., I987. El pensamiento del profesor, vínculo entre la teoría y la práctica, Revista de Educación, Vol. 284, pp. 199-221.

PÉREZ SERRANO,M. 1988.Laformación prácticadel maestro, pp. 103-108. (Escuela Española: Madrid).

PESSOA DE CARVALHO, A.M. (coord.), 1991. Síntesis evolutiva de investigaciones en Enseñanza de Ciencias. Enseñanza de las Ciencias, Vol. 9 (2), pp. 169-174.

RODRIGO, M., UNAMUNO, M., AGRA-CADARSO, M.J., GÓMEZM.A., VIDAL, M.P., MORCILLO,J,G., MARTIN, M., GARCÍA-COLLANTES, M.A. y SILVÁN, E., $1992 \mathrm{a}$. La visión de los alumnos de Magisterio sobre las características del profesor de Ciencias de EGB. Ponencia presentada en VII Congreso de la Asociación Internacional para la Investigación de la Personalidad del Docente (AIRPE). Facultad de Pedagogía. Universidad de Salamanca.

RODRIGO, M., UNAMUNO, M., AGRA-CADARSO, M.J., GÓMEZ, M.A., VIDAL, M.P. y MORCILLO, J.G., 1992b. Algunos aspectos del pensamiento del profesor de Ciencias de EGB. Ponencia presentada en VII Congreso de la AIRPE. Facultad de Pedagogía. Universidad de Salamanca.

ROBERTS, D.A. y CHASTKO, A.M., 1990. Absortion, Refraction, Reflection: An Exploration of Beginning Science Teacher Thinking, Science Education, Vol. 74(2), pp. 197-224.

VILLAR ANGULO, L.M., 1992. Teorias implícitas de los profesores sobre el cambio educativo. Ponencia presentada en VII Congreso de AIRPE. Facultadde Pedagogía. Universidad de Salamanca.

YAGER, E.R. y PENICK, J.E., 1984. What Students say abont science teaching and science teachers, Science Education, Vol. 68(2), pp. 143-152. 


\section{APÉNDICE}

\section{COMPETENCIAS Y CARACTERÍSTICAS ESTUDIADAS}

\section{Competencias relacionadas con el área}

1. Conocer aceptablemente las disciplinas que corresponden al Ârea de Ciencias (Física, Química, Geología y Biología). Esto supondría:

a) Conocer bien los contenidos que se imparten en estas disciplinas hasta un nivel de COU.

b) Ser capaz de leer con facilidad revistas de divulgación científica como Investigación y Ciencia o Mundo Científico.

c) Ser capaz de responder o preparat respuestas adecuadas a todas las dudas que presenten los alumnos en estas disciplinas.

d) Ser capaz de preparar preguntas e interpretar datos relacionados con las disciplinas científicas.

\section{Enseñar las implicaciones sociales de la Ciencia. Esto supondrfa:}

a) Enseñar la relación que tienen las ciencias experimentales con temas como la crisis energética, la contaminación ambiental, la ingeniería genética o la desertización del suelo.

b) Aprovechar acontecimientos sociales de actualidad para ensenar las implicaciones científicas que pueden tener.

\section{Enseñar de manera interdisciplinar. Esto supondría:}

a) Relacionar con frecuencia los aspectos físicos, químicos, biológicos y geológicos de los fenómenos naturales y de la tecnología.

b) Relacionar con frecuencia los aspectos científicos de su asignatura con materias como geografía, historia o filosofía y matemáticas.

c) Cuidar en su enseñanza la expresión oral y escrita de los estudiantes.

4. Enseñar las ciencias como algo cercano a nuestra vida diaria. Esto supondría:

a) Enseñar implicaciones en las ciencias experimentales en temas próximos a los alumnos, como la producción de alimentos, la contaminación ambiental, los animales domésticos y de labor, los automóviles, los electrodomésticos, el cultivo de las plantas, la salud, etc.

b) Promover la aplicación de los métodos de la ciencia en problemas cotidianos.

c) Interpretar, a la luz de los conceptos científicos, fenómenos de la vida diaria.

\section{Enseñar la historia y la filosofia de la ciencia. Esto supondria:}

a) Enseñar las aportaciones que hicieron a la ciencias, en el contexto de su época, científicos como Newton, Lavoisier, Pasteur, Mendel, Darwin. b) Enseñar que los conceptos científicos tienen historia y evolucionan.

c) Enseñar que la mentalidad científica evoluciona al mismo tiempo que la cultura propiamente científica.

\section{Enseñar los métodos de la ciencia. Esto supondría:}

a) Enseñar a observar y medir.

b) Enseñar a distingtiir entre observaciones y suposiciones.

c) Enseñar a comunicar los resultados de observaciones y medidas.

d) Enseñar a obtener conclusiones a partir de unos datos experimentales.

e) Enseñar a diseñar experimentos.

f) Enseñar a trabajar sistemáticamente y con orden.

g) Enseñar a criticar los resultados.

7. Preparary organizar adecuadamente el trabajo de laboratorio. Esto supondría:

a) Preocuparse de recopilar bibliografía sobre prácticas de laboratorio.

b) Enseñar el manejo de aparatos básicos como un microscopio o un voltímetro.

c) Enseñar a recoger datos adecuadamente en tablas y a representarlos gráficamente.

d) Enseñar a utilizat con cuiđado el material de laboratorio.

\section{Competencias relacionadas con las técnicas de enseñanza}

\section{Planificar la enseñanza. Esto supondría:}

a) Formular los objetivos que los alumnos deben alcanzar.

b) Consultar bibliografía diversa para organizar los contenidos de la manera más adecuada.

c) Preparar con antelación las actividades que los alumnos van a realizar en el período de clase.

9. Presentar las ideas a los alumnos de una manera organizada. Esto supondria:

a) Organizar las actividades de aprendizaje de forma que exista una secuencia lógica.

b) Organizar los trabajos prácticos de forma que estén coordinados con los fundamentos teóricos.

c) Hacer con frecuencia introducctones y resúmenes al principio y al final de cada lección.

d) Hacer con frecuencia indicaciones para resaltar la importancia de los puntos más interesantes.

e) Expresarse claramente. 
10. Tener y usar conocimientos de psicología del aprendizaje en la ciencia. Esto supondría:

a) Acomodar la ensefianza de conceptos y destrezas científicas a los estadios que contempla la teoría de Piaget.

b) Organizar los contenidos de acuerdo con alguna teoría del aprendizaje.

c) Partir de los intereses de los alumnos para organizar las actividades de enseñanza.

d) Descubrir errores conceptuales en los alumnos y tratar de corregirlos.

11. Enseñar a través de métodos activos que hagan pensar al alumno y aprender por sí mismo. Esto supondría.

a) Organizar la enseñanza de modo que la clase magistral ocupe como máximo el $50 \%$ del tiempo destinado a la asignatura.

b) Hacer que los alumnos realicen con frecuencia trabajos individuales o en grupo.

c) Establecer diálogo frecuente en clase con los alumnos (realizar cálculos, registrar observaciones, sintetizar una exposición, etc.).

d) Plantear interrogantes y preguntas abiertas.

e) Cuando los alumnos contestan a las preguntas, solicitar con frecuencia explicaciones aclaratorias o que elaboren más las respuestas.

12. Crear un clima humano entre los alumnos que ayude a aprender. Esto supondría:

a) Preocuparse por las dificultades de aprendizaje de los alumnos.

b) Atender con amabilidad las preguntas y dudas de los alumnos.

c) No ridiculizar al alumno que no sabe.

d) Animar a los estudiantes durante la enseñanza.

13. Usar recursos y técnicas de enseñanza diversas. Esto supondría.

a) Organizar la enseñanza de forma que se trabaje en el aula parte del tiempo en gran grupo, en pequeños grupos y con cada alumno individualmente.

b) Utilizar con frecuencia medios audiovisuales como películas, diapositivas o trasparencias.

c) Hacer que los alumnos trabajen con libros diversos.

d) Utilizar en clase los recursos humanos naturales y tecnológicos que le ofrece su entorno.

e) Realizar salidas a] entorno.

f) Construir aparatos y diseffar experimentos de laboratorio por uno mismo.

\section{Enseñar de manera individualizada. Esto supondria:}

a) Asignar tareas diferentes a los alumnos en función de sus conocimientos y posibilidades.

b) Organizar y asignar tareas de recuperación específicas para cada alumno.

c) Permitir que los alumnos trabajen sobre temas que les interesen personalmente.
15. Evaluar adecuadamente el progreso de los alumnos. Esto supondría:

a) Usar diversos instrumentos de evaluación (pnuebas de respuesta múltiple, entrevistas personales, resolución de problemas, etc.) construidos para medir un amplio conjunto de capacidades (conocimientos, comprensión, capacidad de análisis, etc.).

b) Usar los resultados de la evaluación para informar a los alumnos de los puntos en que tienen dificultades de aprendizaje.

16. Usar los resultados de la evaluación como información sobre la efectividad de la enseñanza para revisarla y modificarla cuando sea necesario. Esto supondría:

a) Revisar los contenidos y métodos de enseñanza de un curso para otro de acuerdo con los resultados de las evaluaciones.

b) Revisar los contenidos y métodos de enseñanza de un curso para otro de acuerdo con las sugerencias que hacen directamente los alumnos sobre las dificultades de los temas.

\section{Características personales}

17. Tener estabilidad emocional y confianza en si mismo. Esto supondría:

a) Ser capaz de reconocer errores delante de los alumnos.

b) En general mostrarse de buen humor delante de los alumnos.

c) Reconocer el desconocimiento de ciertos temas.

d) Aceptar de buen grado las críticas se sus alumnos.

\section{Tener sentido del humor. Esto supondria.}

a) Hacer en ocasiones comentarios divertidos sobre los temas que se está enseñando.

b) Aceptar de buen grado las bromas de los alumnos.

19. Mostrar entusiasmo por la materia y por la enseñanza. Esto supondría:

a) Exponer los temas en clase de un manera entusiasta.

b) Estar con frecuencia disponible para ayudar a sus alumnos, fuera de clase, en temas de interés para ellos.

c) Animar a los alumnos a interesarse por temas científicos.

20. Relacionarse cordialmente con padres y alumnos. Esto supondría:

a) Recibir de buena gana la visita de los padres para tratar de sus hijos.

b) Charlar fuera del auta con los alumnos de temas extraacadémicos.

21. Ser autocriticos, reconocer las limitaciones como profesor y evaluarse a sí mismo. Esto supondría:

a) Estar abierto a las aportaciones que se puedan dar en cursos o seminarios para modificar los métodos de enseñanza.

b) Pensar que muchas de las deficiencias en el aprendizaje de los alumnos son debidas a que los métodos de enseñanza no son adecuados.

c) Reflexionar al término de cada curso sobre los errores cometidos. 


\section{Características profesionales}

22. Cooperar con otros profesores. Esto supondria:

a) Poner a disposicion de los colegas los materiales y conocimiento.

b) Reunirse frecuentemente con los profesores del área para organizar ta enseñanza.

c) Reunirse con los profesores de otras áreas para relacionar la enseñanza de unas asignaturas con otras.
23. Perfeccionarsecientífica y pedagógicamente. Esto supondría:

a) Leer revistas científicas.

b) Asistir a cursos sobre temas científicos.

c) Leer revistas educativas.

d) Asistir a cursos sobre temas pedagógicos.

e) Trabajar en actividades de renovación pedagógica.

f) Leer libros científicos. 